\title{
Análisis Praxeológico de los Métodos de Enseñanza: un Puente entre la Investigación y la Práctica
}

\author{
Análise Praxeológica de Métodos de Ensino: uma Ponte \\ entre Pesquisa e Prática
}

\section{Praxeological Analysis of Teaching Methods: A Bridge Between Research and Practice}

\author{
Tobias Espinosa Brasil \\ Ives Solano Araujo Brasil \\ Eliane Angela Veit ${ }^{(1)}$ Brasil
}

Es deseable que los buenos resultados obtenidos en las investigaciones sobre la utilización de los métodos activos de enseñanza sean alcanzados en la práctica docente en diversos contextos. Para ello, es necesario comprender las características específicas de los métodos de enseñanza, así como las eventuales transformaciones necesarias para adecuarlos a diferentes contextos. En este artículo proponemos utilizar el constructo de "Organización Praxeológica", proveniente de la Teoría Antropológica de lo Didáctico, como herramienta para el estudio de los métodos de enseñanza. Desde esa perspectiva, los métodos son vistos como conocimientos institucionalizados - social e históricamente legitimados - que pueden ser estudiados en términos de praxis (tipos de tareas y técnicas) y del logos (discursos tecnológicos y teóricos). Ilustramos estas ideas con la construcción de una organización praxeológica para el método conocido como Team-Based Learning.

Palabras Clave: Teoría Antropológica de lo Didáctico; Organización Praxeológica; Herramienta Analítica; Métodos de Enseñanza.

É desejável que os bons resultados obtidos em pesquisas com métodos ativos de ensino sejam alcançados na prática docente em diversos contextos. Para tanto, é necessário compreender os métodos de ensino em suas especificidades, assim como as eventuais transformações necessárias para adequá-los a diferentes contextos. Neste artigo propomos o uso da Organização Praxeológica, oriunda da Teoria Antropológica do Didático, como ferramenta para o estudo de métodos de ensino. Nessa perspectiva, os métodos são vistos como conhecimentos institucionalizados - social e historicamente legitimados - que podem ser estudados em termos de práxis (tipos de tarefa e técnicas) e do logos (discursos tecnológicos e teóricos). Ilustramos essas ideias com a construção de uma organização praxeológica para o método Team-Based Learning. 
Palavras-chave: Teoria Antropológica do Didático; Organização Praxeológica; Ferramenta analítica; Métodos de ensino.

It is desirable that the good results obtained in research on the use of active teaching methods be achieved in teaching practice in various contexts. To this end, it is necessary to understand the teaching methods in their specificities, as well as the possible transformations necessary to adapt them to different contexts. In this paper, we propose the use of the "Praxeological Organization" construct, grounded in the Anthropological Theory of Didactics, as an analytical tool for the study of teaching methods. In this perspective, the methods are seen as institutionalized knowledge socially and historically legitimated -, which can be studied in terms of praxis (types of tasks and techniques) and logos (technological and theoretical discourses). We illustrate our theoretical proposal with the establishment of a praxeological organization for the teaching method known as Team-Based Learning.

Keywords: Anthropological Theory of Didactics; Praxeological Organization; Analytical tool; Teaching Methods; Team-Based Learning.

\section{Introducción}

Uno de los principales desafíos de la investigación en Educación en Ciencias ha sido reducir la brecha entre investigación y práctica docente (e.g. McIntyre, 2005; Miretzky, 2007; El-Hani, \& Greca, 2012; Tibaud, 2017). Por un lado, muchos investigadores tienen la percepción de que los profesores no utilizan el conocimiento generado por la investigación para construir y reflexionar sobre su práctica; por otro lado, hay profesores que perciben el saber producido en el ambiente académico como distante o ajeno a su realidad.

Es así como ciertos métodos activos de enseñanza - entendidos como aquellos que promueven el compromiso activo y colaborativo de los estudiantes en el proceso de enseñanza y aprendizaje - son ejemplos de conocimientos generados y evaluados en investigaciones empíricas, que enfrentan dificultades para ser apropiados e incorporados a la práctica docente (Khatri et al., 2017). Casos ilustrativos, como Peer Instruction (Mazur, 2015), o Just-in-Time Teaching (Novak, Patterson, Gabrin, \& Christian, 1999) o Team-Based Learning (Michaelsen, Knight, \& Fink, 2004) presentan resultados empíricos positivos, principalmente para la enseñanza de las ciencias a nivel universitario (e.g. Hake, 1998; Crouch, \& Mazur, 2001; Rudolph, Lamine, Joyce, Vignolles, \& Consiglio, 2014). Sin embargo, para ser transferidas a la realidad escolar de instituciones ${ }^{1}$ distintas a las de origen, los métodos sufren modificaciones que son, en parte, debidas al cambio de contexto epistemológico (Chevallard, 1991). Esas modificaciones pueden ser

1 En todo el artículo, utilizamos el término institución en un sentido amplio, que puede englobar tanto locales físicos, como escuelas y universidades (sentido tradicional), o cualquier agrupación social legitimada, sea una familia, un juego, una clase. La autoridad legitimadora puede ser personal, un padre, un juez, un profesor; o una autoridad difusa, basada en la concordancia en torno a algunos principios (Douglas, 1986; Chevallard, 2000). 
consideradas positivas cuando potencian las ideas originales del método, expandiéndolas para contemplar un contexto inédito, o, dicho de otra forma, cuando el conocimiento académico es incorporado a una nueva situación didáctica (Rogers, 2003; Henderson, \& Dancy, 2008); y negativas cuando se realizan simplificaciones indebidas que desvirtúan las intenciones originales del método. A menudo, estas situaciones se dan por presiones institucionales fuertemente cimentadas en un paradigma de enseñanza tradicional, en el cual los alumnos, como si visitasen una obra o exposición, reciben pasivamente las informaciones, sin cuestionarlas y ajenos a su construcción social (Chevallard, 2013).

No es raro entonces que métodos activos de enseñanza sean interpretados como mero conjunto de técnicas a disposición del profesor, contribuyendo a una actuación docente de tipo racionalista técnica, cuya "práctica profesional consiste en la solución instrumental de problemas mediante la aplicación de un conocimiento teórico y técnico, previamente disponible, que procede de la investigación científica" (Contreras, 2012, p. 101). Sin embargo, además de estar constituidos por técnicas, los métodos también están compuestos por discursos que, a pesar de no siempre ser explícitos, legitiman tales técnicas.

Por ejemplo, el método de Peer Instruction está, en líneas generales, compuesto por técnicas como la votación de respuestas a cuestiones conceptuales y la discusión entre pares de esas respuestas, sustentadas por el argumento discursivo que asocia la promoción del aprendizaje conceptual a la interacción entre los alumnos. En clase, el profesor, después de una breve exposición oral, presenta a los estudiantes una cuestión conceptual con respuestas de elección múltiple. Cada alumno responde a la cuestión individualmente utilizando un sistema de votación (e.g. Flashcards o Clickers). Dependiendo de la cantidad de respuestas correctas (de 30 a 70\%) los alumnos son orientados a discutir y convencer a sus colegas de sus respuestas. A continuación, una nueva votación es realizada y, a partir de la nueva distribución de respuestas, el profesor decide si continúa con un nuevo tópico o retoma el contenido con una nueva pregunta conceptual (Araujo, \& Mazur, 2013; Mazur, 2015). Esta praxis de Peer Instruction se basa en la idea de que el intercambio de informaciones entre los alumnos es esencial para la construcción del conocimiento y que la acción de enseñar o aprender con un colega complementa y amplía la enseñanza del profesor. En ese sentido, una técnica como la votación ya mencionada, además de brindar un feedback al profesor acerca de la comprensión de los estudiantes sobre los conceptos-clave en estudio, lo ayuda a orientar las discusiones entre pares. Pero, al ser transferida esta propuesta a una institución en que la pedagogía dominante está asociada a una visión transmisiva del conocimiento, esa misma técnica puede ser reinterpretada como una manera de evaluar la recepción adecuada del mensaje transmitido por el profesor. En ese enfoque, una baja frecuencia de aciertos puede ser entendida por el profesor como una falla en la transmisión del conocimiento, llevándolo a substituir la técnica subsecuente, que originalmente es la discusión entre pares, por exposiciones orales más detalladas. Ese cambio indebido claramente desvirtuaría el método, por tratarse de una modificación negativa realizada 
en función de creencias sobre la enseñanza y el aprendizaje dominantes en la institución.

Disponer de una herramienta analítica que ayude al investigador a identificar discursos que legitiman las técnicas y tareas propuestas puede ser útil para analizar la coherencia de las acciones realizadas en la práctica a la luz de los referenciales teóricos, así como investigar las prácticas generadas y sus transformaciones cuando son implementadas en contextos educacionales particulares. De esa forma se puede contribuir tanto a la articulación entre teoría y práctica, como a la construcción de un puente entre investigación empírica con métodos de enseñanza activos y la práctica educativa. Para ello proponemos la Teoría Antropológica de lo Didáctico (TAD) como contribución a la creación, implementación y evaluación de los métodos de enseñanza. En particular, utilizaremos el concepto de Organización Praxeológica, asumido como un modelo que describe cualquier actividad humana, social e históricamente legitimada, que se realiza regularmente en términos de prácticas y discursos que los legitiman (Chevallard, 1999, 2019).

El objetivo principal de este trabajo es presentar la noción de Organización Praxeológica, sustentada en la TAD, como herramienta analítica útil para el análisis de métodos de enseñanza, considerándolos como cuerpos de conocimientos - constituidos por tareas, técnicas, tecnologías y teorías - que sufren transformaciones en el paso a nuevos ámbitos institucionales.

Para ejemplificar nuestra propuesta presentaremos un análisis del método de enseñanza conocido como Team-Based Learning en su propuesta original (Michaelsen, et al., 2004).

\section{Marco teórico}

La perspectiva de la antropología didáctica surge, en parte, como una respuesta a las críticas a la Teoría de la Transposición Didáctica. Las principales críticas, que podrían poner en duda nuestro emprendimiento al tratar de aproximar la investigación y la práctica, son: la idea de que el conocimiento racional (el producido por los investigadores) es superior al saber que se debe enseñar (presente en los planes de estudio y los libros de texto) y conocimiento enseñado en el aula; y la concepción del profesor como un simple reproductor de conocimiento producido en otras instancias de la sociedad (Chervel, 1990; Caillot, 1996; Cardelli, 2004).

En relación a una supuesta jerarquía de conocimiento, Winsløw (2011) aclara que el enfoque de la transposición didáctica no es simplemente el transporte de conocimiento de varias fuentes para "servir" a los estudiantes; es, sobre todo, el análisis profundo de los cambios que experimenta un conocimiento cuando transita interinstitucionalmente. Lo que Chevallard pone en evidencia es la diferencia cualitativa entre el conocimiento, que está inmerso en las instituciones que los legitiman, con condiciones ecológicas únicas, y no existe una jerarquía entre ellos (Marandino, 2016). En cuanto al papel del docente, Leite (2004) enfatiza que, aunque Chevallard no otorga exclusividad al docente en el sistema educativo, o incluso una prioridad, argumenta que el docente puede participar 
tanto en la transposición de conocimientos como en la creación de nuevos saberes. Consideramos las críticas mencionadas ya superadas y compartimos las interpretaciones de los autores mencionados.

La Teoría Antropológica de lo Didáctico (TAD) propuesta originalmente en el campo de la enseñanza de la Matemática centra el estudio en el conjunto de las actividades humanas y de las instituciones sociales. Parte del axioma de que un individuo "no es, en la realidad, más que la emergencia de un complejo de sujeciones institucionales" (Chevallard, 2000, p. 132); la libertad individual se da pues en el conflicto entre esas sujeciones. Desde esta perspectiva, la comprensión de la actividad humana - de por qué ella es como es - depende de la comprensión de cómo viven y se desarrollan las instituciones en las que ocurre la actividad.

Imbricado a las instituciones está lo que Chevallard (1999, 2000, 2019) llama sistema didáctico, compuesto por uno o varios sujetos que ocupan una posición de estudiante, uno o varios sujetos que ocupan la posición de profesor y una cuestión a ser estudiada. Los cambios en las relaciones entre los sujetos están a menudo vinculados a una intención institucional, la cual es alterada por esas relaciones personales, que, a su vez, están antropológicamente correlacionadas con el surgimiento de intenciones didácticas (Chevallard, 2000).

Para la TAD, el fenómeno didáctico, y toda actividad humana, puede organizar bajo un único modelo, llamado praxeología.

\section{Organizaciones Praxeológicas}

Chevallard (1999) propone que todas las actividades humanas que se realizan regularmente, legitimadas social e históricamente, pueden describirse a través de un modelo llamado Organización Praxeológica (OP), que consta de dos niveles: saber hacer (praxis) y saber (logos). El primer nivel se expresa en los tipos de tareas [T] y técnicas $[\tau]$ para resolverlos, mientras que los logos están compuestos por tecnologías $[\theta]$, entendidas en el contexto de la Teoría Antropológica de lo Didáctico desde la etimología de la palabra, como los discursos para justificar, explicar y generar las técnicas y las teorías $[\Theta]$ que constituyen discursos que, con un mayor grado de profundidad, justifican, explican y generan las tecnologías.

Las tareas están en la raíz de la praxeología (Chevallard, 1999). Para cada tipo de tarea $\mathrm{T}$, varias tareas $\mathrm{t}$ pueden estar asociadas, las cuales pueden variar en grado de especificidad. En general, Tareas y Tipos de Tareas son expresadas por un verbo: caminar, ejecutar, reproducir, escribir, calcular, etc. Chevallard alerta sobre tres puntos importantes:

1. Enla Teoría Antropológica delo Didáctico la noción de tarea tiene connotación más amplia que en el lenguaje ordinario. "Rascarse la mejilla, ir del sofá al armario, y hasta sonreír son también tareas" (Chevallard, 1999, p. 222)

2. La noción de Tipo de Tarea empleada en la teoría presupone un determinado grado de precisión. "Calcular el volumen de una esfera" o "Escribir una 
redacción” son Tipos de Tarea, pero "Calcular" o "Escribir” no lo son. Estas dos últimas son géneros de tareas.

3. Por último, los elementos géneros, tipos de tareas y tareas son construcciones institucionales y no datos de la naturaleza.

Veamos un ejemplo de género, Tipo de tarea y Tarea para la Física. "Calcular" se puede considerar un género de tarea, mientras que "calcular la magnitud del campo eléctrico en las proximidades de las distribuciones de carga simétricas" es un tipo de tarea. A cada tipo de tarea $\mathrm{T}$ pueden estar asociadas diversas tareas $\mathrm{t}$, que varían en el grado de especificidad, como, por ejemplo: "calcular la magnitud del campo eléctrico de un cable largo uniformemente cargado en un punto P próximo a él”.

Las técnicas $[\tau]$ constituyen maneras de resolver una tarea. Las técnicas tienen alcance limitado, y permiten resolver una cantidad específica de tareas. Ellas no pueden ser confundidas con algoritmos, los cuales son tipos particulares de técnicas. Sólo en casos excepcionales una técnica es un algoritmo. Normalmente, la aplicación de una técnica presupone cierto grado de indeterminación, aún cuando ella esté bien definida (Chevallard, Bosch, \& Gascón, 2001).

Para resolver la tarea t expuesta anteriormente una técnica posible es la aplicación de la Ley de Gauss para la electricidad. Otra técnica posible sería considerar elementos infinitesimales de carga y utilizar el principio de superposición, calculando la magnitud del campo eléctrico en el punto $\mathrm{P}$ a partir de la Ley de Coulomb.

Una tarea $t$ puede ser resuelta con diferentes técnicas - algunas tienen alcance y precisiones mayores que otras. En ese sentido, es posible establecer relaciones entre técnicas y establecer un grado de superioridad entre ellas.

Para la institucionalización de una técnica, ésta debe ser compresible $y$, principalmente, justificable. La existencia de una técnica presupone la existencia subyacente de una tecnología (de tekhné, técnica, y logos, discurso), o sea, de un discurso racional que justifica, explica o genera la técnica. La palabra tecnología, diferente del significado de sentido común, es empleada por Chevallard en su sentido etimológico.

La tecnología $[\theta]$, así como los otros elementos de la OP, puede ser reconocida en una institución y no en otra. Además, el discurso tecnológico en torno de una técnica en una cierta institución puede ser transferido para otra institución. La ecología de la nueva institución puede transformar la tecnología original, adaptándola a una nueva realidad, cuya praxis y logos ya están bien establecidas. El conocimiento acerca de nuevos discursos, así como de nuevas técnicas, puede darse por medio de canales de comunicación en masa (e.g. libros, artículos y páginas de internet) o interpersonales (e.g. reuniones, seminarios y grupos de estudio). De acuerdo a Rogers (2003) la comunicación en masa es eficiente para que un gran número de personas conozca una nueva idea; ya los canales interpersonales facilitan el proceso de persuasión para que una nueva idea sea adoptada.

La tecnología que justifica el uso de la Ley de Gauss como técnica para determinar el campo eléctrico de un hilo largo y cargado, se basa en que los cálculos resultan más 
simples en situaciones donde las líneas de campo eléctrico tienen alto grado de simetría. Otro discurso tecnológico, que en este caso explica la técnica, es que el flujo eléctrico de una superficie gaussiana es igual a la carga neta en su interior.

El mismo rol que las tecnologías ejercen en relación a las técnicas, las teorías $[\Theta]$ ejercen en relación a las tecnologías. O sea, una teoría justifica, explica o genera una o más tecnologías. Ella constituye un grado superior de discurso de justificación, explicación o construcción (Chevallard, 1999), que no siempre está explícito en las descripciones de las actividades humanas. Volviendo a nuestro ejemplo, la teoría que justifica, explica o genera el discurso tecnológico relativo a la Ley de Gauss es la Teoría Electromagnética.

El bloque teórico-tecnológico es el que permite la preservación de las prácticas y su comunicación con los otros, para que también puedan participar en su construcción.

$\mathrm{Al}$ ser transpuestos de un contexto epistemológico a otro, las OPs pueden sufrir transformaciones (Chevallard, 1991; Winsløw, 2011). Por ejemplo, el conocimiento desarrollado por los investigadores en física (saber sabio) sufre modificaciones para, a partir de presiones institucionales externas a la escuela, convertirse en conocimiento que el profesor enseña en la escuela (saber a ser enseñado), usualmente presente en los libros didácticos. El saber a ser enseñado, a su vez, al ser transferido a la sala de aula, pasa por una segunda transformación (transposición interna) que genera la OP del saber enseñado.

En la Teoría Antropológica de lo Didáctico esas transformaciones no ocurren sólo con los saberes físicos o matemáticos, sin con cualquier actividad humana cualquier Organización Praxeológica. A medida que surgen nuevas tareas, las OPs pueden envejecer y tornarse obsoletas. Así, pueden nacer nuevas praxeologías dentro de una determinada institución I, producidas en la propia institución o importadas de otra institución I'. Normalmente, las condiciones ecológicas impuestas por I no permiten que una praxeología proveniente de I' sea reproducida de forma idéntica.

Chevallard sostiene que el trabajo de transposición puede mejorar una praxeología y expandir aquellas socialmente disponibles, ya que se adapta a condiciones institucionales sin precedentes. En sus palabras:

(...) en materia de transposición didáctica, por ejemplo, es decir, cuando I es una institución didáctica (una escuela, una clase...), sucede con frecuencia, sobre todo cuando I' no es una institución sabia, que el trabajo transpositivo sea la ocasión de mejorar la praxeología así vuelta a trabajar - simplificándola, precisando algunos de sus elementos, etc. En todo caso, la transposición enriquece el mundo de las praxeologías socialmente disponibles- en la medida en la que crea una praxeología adaptada a ciertas condiciones institucionales inéditas. (Chevallard, 1999, p. 228).

Para analizar una transposición didáctica, se puede utilizar/crear un Modelo Epistemológico de Referencia, desde el cual es posible "deconstruir y reconstruir las praxeologías cuya difusión intrainstitucional e interinstitucional pretende analizar" (Gascón, 2011, p. 208). Gascón (2011) enfatiza que este modelo es siempre provisional y debe ser revisado constantemente. Además, no existe un sistema de referencia 
privilegiado para analizar cada etapa de la transposición didáctica (Bosch y Gascón, 2006; Gascón, 2011). El modelo de referencia puede basarse en el saber científico, si es de interés del investigador analizar cómo se transforma este saber; o puede ser saber escolar, ya sea creado por el profesor, el investigador o el profesor-investigador.

Pasamos a argumentar como los métodos de enseñanza pueden ser comprendidos como una organización praxeológica y ser analizados a la luz de la antropología didáctica de Chevallard.

\section{Los métodos de enseñanza como organizaciones praxeológicas}

Las investigaciones en el marco de la Teoría Antropológica de lo Didáctico presentan enfoques diferentes, aunque la mayoría de ellas utiliza la Organización Praxeológica como herramienta analítica. Las praxeologías se utilizan, principalmente, en el análisis del material didáctico (e.g. Schivani, Brockington, \& Pietrocola, 2013; Barbé, Espinoza, \& Gellert, 2017; Wijayanti, \& Winsløw, 2018; Wijayanti, 2019) y de la práctica en el aula (e.g. Barbé, Bosch, Espinoza, \& Gascón, 2005; Corica, \& Otero, 2011, 2019). También son utilizadas en el diálogo entre teorías (networking theories) (e.g. Trigueros, \& Martínez-Planell, 2015; Bosch, Gascón, \& Trigueros, 2016) y en el campo de la enseñanza de las ciencias en museos (e.g. Achiam, 2013; Marandino, Bueno, Kristel, \& Oliveira, 2016; Bueno, \& Marandino, 2017; Achiam, Lindow, \& Simony, 2019). En el análisis de los materiales didácticos se destacan los elementos de una Organización Praxeológica, sea ella de Física, Matemática o Didáctica, que están presentes en el material escrito, con el objetivo de una mejor comprensión. Por medio de observaciones y entrevistas son construidas praxeologías que describen la estructura de la práctica del aula. En los diálogos, las teorías científicas son consideradas praxeologías de investigación, o sea, están constituidas por tareas, técnicas, tecnologías y teorías. En esta visión epistemológica, los diálogos toman como punto de partida: los tipos de problemas, los elementos teóricos y el componente metodológico (incluyendo las técnicas y tecnologías) de cada teoría. Finalmente, en la enseñanza en el ámbito de los museos, las praxeologías son utilizadas para comprender las transformaciones que se producen en el saber al ser transferido a exposiciones en museos de ciencias.

En particular, los resultados obtenidos en el análisis de los libros didácticos, en la práctica en el aula y en las transposiciones praxeológicas de la didáctica en el contexto de los museos, nos permiten vislumbrar la potencialidad de las Organizaciones Praxeológicas para esta nueva aplicación que proponemos: el análisis de los métodos de enseñanza en su diseño original, la planificación de implementaciones en determinados contextos y sus efectivos usos en el aula.

Generalmente, las investigaciones en el marco de la Teoría Antropológica de lo Didáctico discuten dos tipos de praxeologías: Organizaciones de Contenido (estructura de un cuerpo de conocimiento institucionalizado) y Organizaciones Didácticas (estructura acerca de cómo el conocimiento es estudiado en una institución). En el proceso de estudio hay dos actores, alumno y profesor, que necesitan cumplir tareas de 
acuerdo a su rol. Es por ello que las Organizaciones Didácticas son presentadas en las dos perspectivas.

En el presente artículo, proponemos el estudio específico de métodos de enseñanza como conocimiento a ser estudiado. O sea, así como podemos estudiar las organizaciones matemáticas y físicas, podemos estudiar la Organización del Método de Enseñanza (OME) (e.g. organización praxeológica de Peer Instruction, Team Based Learning, etc.). A partir de diferentes organizaciones didácticas, es posible aislar elementos comunes que corresponden al método de enseñanza adoptado (Figura 1). O sea, hay diferentes Organizaciones Físicas (OF), sobre el saber físico a ser enseñado (u otro saber de referencia), y distintas Organizaciones Didácticas (OD) relacionadas, sin embargo todas ellas pueden ser organizadas con el mismo método de enseñanza. Obviamente, la elección de un método de enseñanza específico interfiere en la OD, así como la OD interfiere en la OME. Por ejemplo, una Organización Didáctica que privilegia el estudio de los aspectos conceptuales de determinado contenido en detrimento de los aspectos matemáticos puede llevar a la institución a adoptar métodos de enseñanza como el de Peer Instruction; de manera análoga, la elección institucional por ese método puede transformar una OD que privilegiaba aspectos matemáticos.

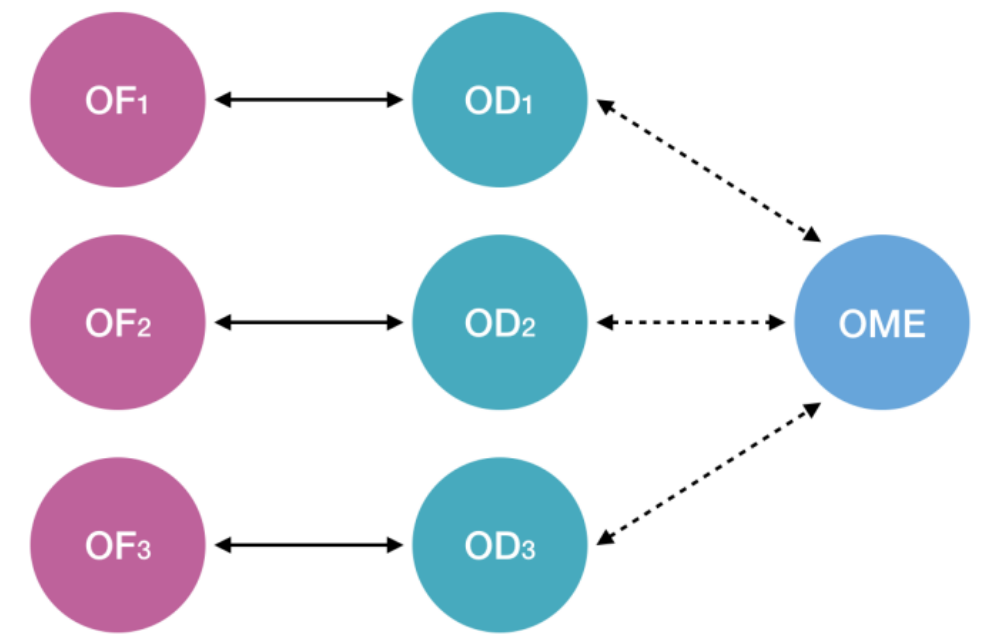

Figura 1. Relaciones dialécticas entre Organización Física (OF), Organización Didáctica (OD) y Organización del Método de Enseñanza (OME)

Además de lo expresado, podemos pensar en organizaciones didácticas de los métodos de enseñanza, las cuales estructuran cómo los métodos son estudiados en una cierta institución.

Entendemos que los métodos de enseñanza constituyen un cuerpo de conocimiento cuya naturaleza es antropológica, social e históricamente legitimada. Utilizando este abordaje consideramos que un método de enseñanza está compuesto por Tipos de Tareas que deben ser realizadas - algunas de ellas por los estudiantes y otras por el profesor -, técnicas, tecnologías que justifican, describen e generan las técnicas, y teorías que justifican, describen y generan las tecnologías. La Figura 2 muestra cómo pueden ser identificados los elementos de un método de enseñanza. 


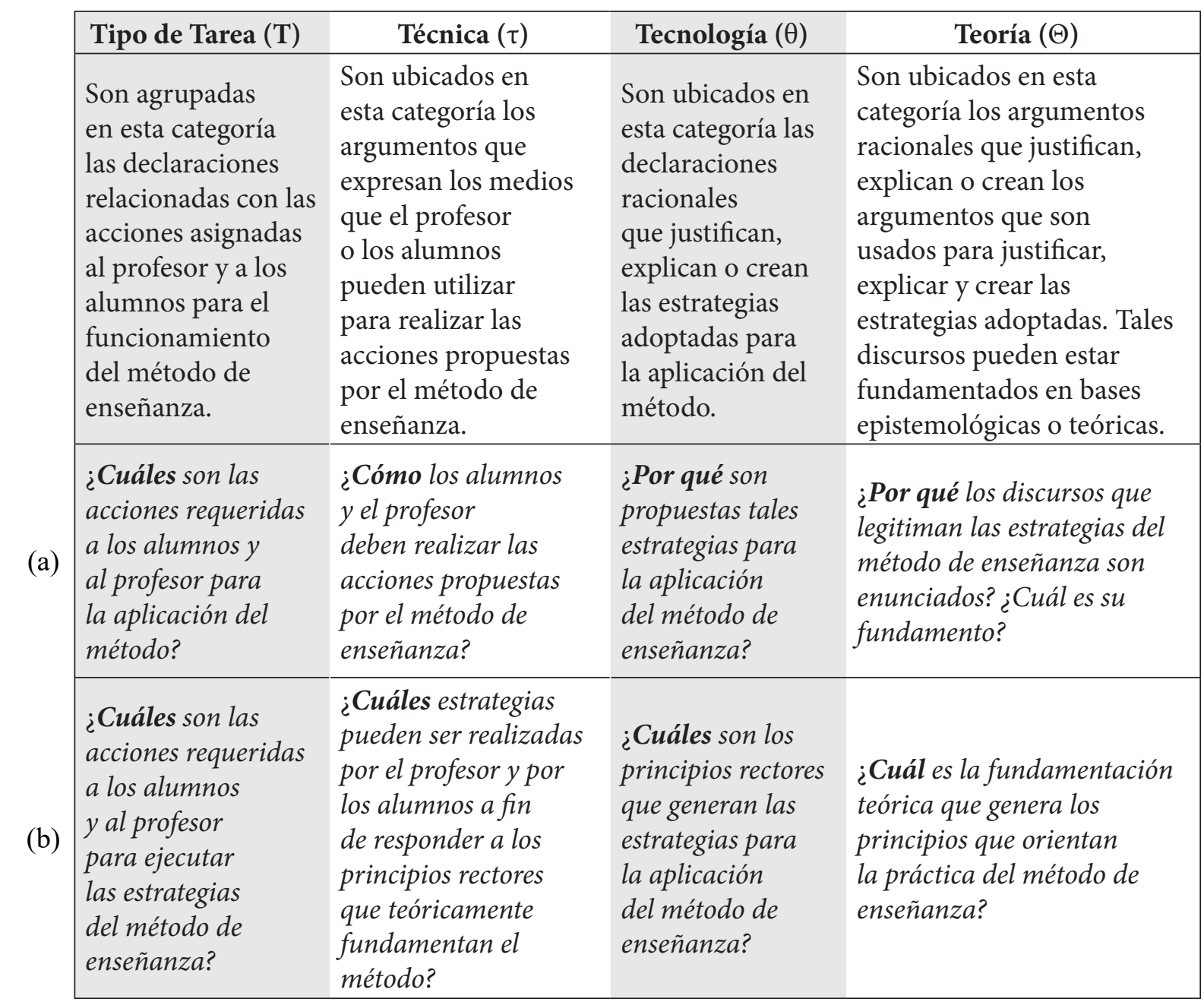

Figura 2. Dos caminos metodológicos posibles para el análisis y la creación de métodos de enseñanza en términos de Tipos de Tareas, Técnicas, Tecnologías y Teorías. (a) camino de análisis a partir de la práctica; (b) camino de creación metodológica a partir de un referencial teórico.

El investigador interesado en analizar a fondo un método exitoso, o que pretenda entender cómo las innovaciones didácticas producidas en la investigación empírica pueden ser adaptadas en la práctica dentro de contextos educacionales particulares, puede dar inicio al análisis con la siguiente pregunta: ¿cuáles son las acciones requeridas a los alumnos y al profesor para la aplicación del método? A partir de eso, puede cuestionarse respecto a cómo los actores realizan las acciones, por qué lo hacen de la manera propuesta y en cuáles razones se fundamentan las mismas. Desde el punto de vista del investigador que quiere construir un método de enseñanza fundamentado teóricamente, uniendo la investigación teórica y la práctica de aula, él puede iniciar con la siguiente pregunta: “¿Cual es la fundamentación teórica que genera los principios que orientan la práctica del método de enseñanza?". A continuación, cuestionarse acerca de los principios rectores, de las estrategias prácticas coherentes y de los tipos de tarea que deben ser realizados por el profesor y por los alumnos. En ambos casos, el investigador tiene sustento para establecer una articulación entre investigación y 
práctica. En el presente artículo nos enfocamos en el análisis minucioso de métodos activos de enseñanza exitosos en el contexto de investigación, pero poco adoptados en la práctica. Es importante resaltar que incluso los métodos creados a partir de la práctica tienen al menos embriones asociadas a las técnicas utilizadas para resolver los tipos de tareas (Chevallard, 1999).

En la metodología de enseñanza Flipped Classroom, por ejemplo, propuesta en el libro escrito por Bergmann y Sams (2012) se encuentra en el topos ${ }^{2}$ del estudiante, el siguiente tipo de tarea: estudiar previamente las aulas. Para realizarlo, se indica la siguiente técnica: asistir a un video propuesto por el profesor, haciendo anotaciones y elaborando preguntas. Un discurso tecnológico que justifica el uso de tal técnica es que el alumno adquiere control sobre su propio aprendizaje, pudiendo estudiar con un ritmo propio. El estudiante puede asistir al video cuantas veces considere necesario, puede hacer pausas, buscar otras fuentes y hacer preguntas específicas al profesor acerca de los puntos en los que tuvo dificultades de comprensión. En el libro, las teorías no están presentes explícitamente.

Los métodos activos de enseñanza muchas veces son presentados con demasiada énfasis en el bloque práctico-técnico. Autores que investigan la adopción de métodos activos de enseñanza apuntan que los profesores raramente ponen en práctica las innovaciones en la forma en que son prescriptas por sus creadores y, normalmente, las modifican reduciendo sus potencialidades. Foote (2016) y Henderson y Dancy $(2009,2010)$ constataron que generalmente los profesores no están conscientes de los principios pedagógicos que sustentan las técnicas de los métodos, lo que dificulta su adopción. Para estos autores, al tomar contacto con las innovaciones didácticas, los profesores necesitan ser alertados sobre tales principios, destacando aquellos elementos del método que son flexibles y aquellos que los modifican. Tales principios pedagógicos forman parte de lo que llamamos el bloque tecnológico - teórico. Además de eso, como destacamos en la introducción, abordar los métodos de enseñanza como conjuntos de técnicas puede dificultar el diálogo entre investigación y práctica, ya que se dificulta la comprensión de la transformación de los métodos de enseñanza cuando transitan interinstitucionalmente.

En contraposición a los métodos activos de enseñanza, otras propuestas didácticas, propias del campo de la Didáctica de las Ciencias y de la Matemática, poseen un bloque teórico-tecnológico bien desarrollado. Por ejemplo, como consecuencia de la Teoría Antropológica de lo Didáctico, se propusieron los Recorridos de Estudio e Investigación como una forma de organización didáctica basada en la construcción de diferentes caminos a partir de una sucesión de preguntas y respuestas articuladas a través de procesos de modelaje (Barquero, Bosch, \& Gascón, 2011).

Considerando la Organización del Método de Enseñanza como una Organización Praxeológica, las transformaciones que ocurren al llevar de una institución a otra pueden ser sintetizadas como en la Figura 3. La Organización del Método de Enseñanza

2 Topos representa el papel (o lugar) de un determinado actor en una actividad humana compleja. 
desarrollada en su contexto de origen, normalmente por investigadores, constituye un saber de referencia, que se acostumbra a exponer en libros o artículos científicos. Esa OME de referencia pasa por la transposición externa, transformándose en la Organización del Método de Enseñanza planificada (OME'). En esta transformación, actúan factores externos al aula en la cual se pretende implementar el método de enseñanza, como por ejemplo políticas públicas, evaluaciones externas, normas de departamento, tiempo, materiales y estructura disponibles en la institución, concepciones sobre la enseñanza y el aprendizaje institucionalizadas y pedagogía dominante. En esencia, las modificaciones resultantes de la transposición externa, por más que usualmente sean realizadas por el profesor al considerar las limitaciones y posibilidades institucionales, son poco influenciadas por él (sus creencias, actitudes, preferencias etc.). Pero, la transposición interna, que genera la transformación de la OME planificada en la Organización de Método de Enseñanza Aplicada (OME”), es impulsada, principalmente, por decisiones del docente al enfrentarse con exigencia de un grupo específico de aprendices (creencias, actitudes, conocimientos, clase social de los aprendices, las relaciones interpersonales desarrolladas en la clase etc.).

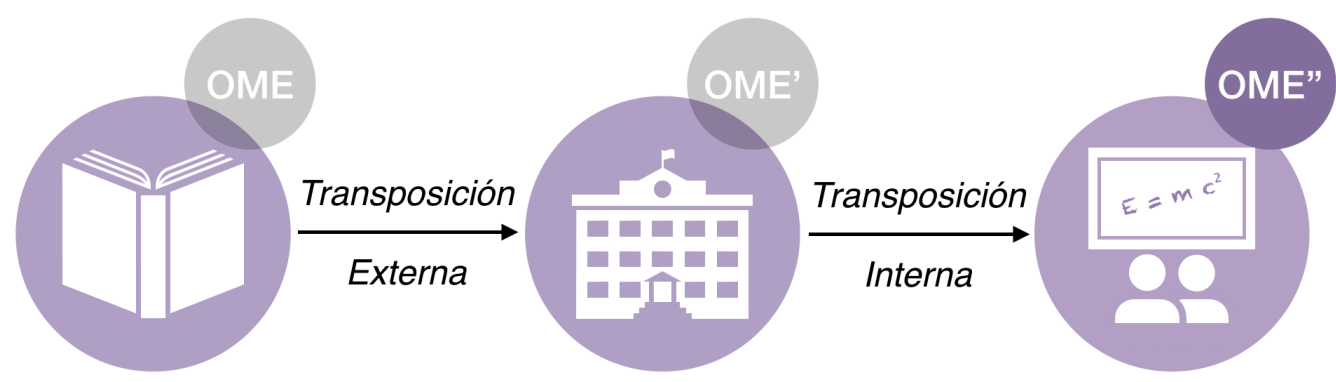

Organización de referencia

Instituciones externas al local de implementación del método de enseñanza (e.g. universidad/escuela de origen del método de enseñanza, grupo de investigación etc.)
Organización planificada

Universidad/escuela a ser implementado el método de enseñanza
Organización aplicada

El aula

Figura 3. Transposición praxeológica de un método de enseñanza (basado en Winsløw (2011))

A continuación, como apoyo al argumento de que las Organizaciones Praxeológicas pueden servir como herramienta analítica para el estudio de los métodos de enseñanza, presentamos la construcción de una organización de referencia para el método de enseñanza Team-Based Learning.

\section{Una Organización Praxeológica de referencia para el método Team-Based Learning}

Para que el lector acompañe el análisis realizado, presentamos una breve 
descripción del método de enseñanza Team-Based Learning (TBL).

El TBL, originalmente descripto por Michaelsen, Fink y Knight (2004), tiene dos objetivos principales: mejorar la comprensión del contenido y desarrollar equipos de aprendizaje. Una disciplina donde se usa TBL se organiza en módulos, con dos fases principales: la preparación y la aplicación. En la primera, los alumnos, antes de la clase, realizan una lectura previa. En la clase, todavía en la fase de preparación, ellos responden, individualmente, a un test de respuestas múltiples referido a los aspectos centrales de la lectura (Test de Preparación Individual). A continuación, el mismo test es respondido en equipo (Test de Preparación en equipo). Cada equipo recibe un cartel (semejantes a aquellos de premio instantáneo) con un valor de respuesta; se discute entre los colegas de equipo intentando lograr un consenso respecto a la alternativa que consideran correcta. Al raspar esa alternativa en el cartel, de inmediato toman conocimiento si la respuesta es o no correcta. En caso negativo, los alumnos vuelven a discutir tratando de entender por qué optaron por la alternativa errada. En caso de tener alguna objeción a una determinada respuesta o a la formulación de la pregunta, los alumnos pueden presentar ese recurso al profesor.

En la fase de aplicación, los estudiantes, en sus casas, individualmente, resuelven una serie de problemas de aplicación de los conceptos estudiados en la fase de preparación. En el aula, resuelven, en equipo, problemas de aplicación más complejos. Los equipos trabajan en un problema por vez y, al final de cada solución, exponen las respuestas para los otros equipos para discutir las diferentes soluciones. Mayores informaciones sobre el Team-Based Learning pueden ser encontradas en Espinosa, Araujo y Veit (2016).

A fin de construir una Organización del Método de Enseñanza de referencia para el TBL, identificamos los principales tipos de tareas del método, en el topos del alumno y en el profesor, las técnicas y los discursos tecnológicos, tales como son descriptos en el libro de Michaelsen, Fink y Knight (2004). El análisis se logró por medio de la lectura integral del libro y la categorización e interpretación a partir de el camino (a) de la Figura 2. Los datos no estaban originalmente organizados, o sea, los autores no presentan las tareas, seguidas de las técnicas y justificaciones. Es por ello que en el análisis se procuró encontrar los elementos en común e interpretarlos a la luz de los componentes de la Organización Praxeológica ${ }^{3}$. En la Figura 4 se expone un ejemplo del proceso de análisis (categorización e interpretación) realizada en el topos del alumno.

La Organización del Método de Enseñanza Team-Based Learning completa, construida en el topos del alumno y en el topos del profesor, puede ser encontrada en la tesis de Espinosa (2019). Aquí se destacan algunas características principales de esta praxeología:

1. La ausencia de teorías explícitas

2. La importancia dada al desarrollo de los equipos y al tiempo necesario detransformación de los grupos en equipos

3 Una vez que la construcción de una Organización Praxeológica depende de la interpretación del investigador, no hay una única praxeología para cada método de enseñanza. 
3. La noción de que la comprensión conceptual es esencial para la resolución de problemas

4. El incentivo a la realización de tareas por medio de los testes evaluados conforme las respuestas sean correctas o erróneas (científicamente).

\begin{tabular}{|c|c|c|}
\hline Pregunta orientadora & Fragmentos del libro & Interpretación \\
\hline $\begin{array}{l}\text { ¿Cuáles son las } \\
\text { acciones requeridas a } \\
\text { los alumnos para la } \\
\text { aplicación del método? }\end{array}$ & $\begin{array}{l}\text { "Las macro-unidades de instrucción del Team- } \\
\text { Based Learning normalmente involucran tres } \\
\text { tipos diferentes de actividades en el aula, cada } \\
\text { una de ellas está precedida por preparación } \\
\text { previa" (p. 36, traducción nuestra, itálica } \\
\text { nuestra). }\end{array}$ & $\begin{array}{l}\mathbf{T}_{1} \text { - Prepararse } \\
\text { previamente para las } \\
\text { clases. }\end{array}$ \\
\hline $\begin{array}{l}\text { ¿Cómo los alumnos } \\
\text { deben realizar las } \\
\text { acciones propuestas } \\
\text { por el método de } \\
\text { enseñanza? }\end{array}$ & $\begin{array}{l}\text { "En la mayoría de los casos, los estudiantes } \\
\text { inicialmente son expuestos a conceptos a } \\
\text { través de lecturas indicadas". (p. 38, traducción } \\
\text { nuestra, itálica nuestra) } \\
\text { "Los alumnos deben completar las lecturas y } \\
\text { llegar a la clase siguiente preparados para hacer } \\
\text { un test sobre el material que acabaron de leer" } \\
\text { (p. 42, traducción nuestra, itálica nuestra). }\end{array}$ & $\begin{array}{l}\boldsymbol{\tau}_{1} \text { - Leer fuera del aula un } \\
\text { material indicado por el } \\
\text { profesor. }\end{array}$ \\
\hline $\begin{array}{l}\text { ¿Por qué son propuestas } \\
\text { tales estrategias para la } \\
\text { aplicación del método } \\
\text { de enseñanza? }\end{array}$ & $\begin{array}{l}\text { "Si los aprendices no realizan individualmente } \\
\text { las tareas previas, no podrán contribuir con su } \\
\text { equipo" (p. } 31 \text {, nuestra traducción). } \\
\text { "[...] cada miembro es explícitamente } \\
\text { responsable de su preparación previa "(p. } 43, \\
\text { traducción nuestra). } \\
\text { "El instructor [...] tiene suficiente tiempo } \\
\text { en clase para permitir a los estudiantes que } \\
\text { se involucren con las tareas de aplicación y } \\
\text { desarrollen habilidades de aprendizaje de alto } \\
\text { nivel" (p. } 43 \text {, traducción nuestra). }\end{array}$ & $\begin{array}{l}\theta_{1} \text { - El primer contacto } \\
\text { extra clase valora el tiempo } \\
\text { en el aula. Además, el } \\
\text { alumno se responsabiliza } \\
\text { por su preparación. } \\
\text { Esta actividad tiene } \\
\text { como objetivo preparar } \\
\text { al estudiante para la } \\
\text { discusión y resolución } \\
\text { de los problemas de } \\
\text { aplicación. }\end{array}$ \\
\hline
\end{tabular}

Figura 4. Ejemplo del proceso de categorización e interpretación de la Organización del Método TeamBased Learning para el topos del alumno

El desarrollo pragmático, a partir de problemas docentes de aula, tal como el Team-Based Learning, es característico de los métodos de enseñanza norteamericanos. En ese sentido, es difícil encontrar teorías explícitas que justifiquen las tecnologías. El desarrollo es concretizado en la práctica y los avances y cambios son realizados a partir de resultados de investigación empírica. Una técnica como la resolución de problemas de aplicación en equipo, por ejemplo, es parcialmente justificada por un buen resultado de investigación: "generalmente, el desempeño en equipo es superior al del mejor integrante del grupo". De acuerdo a Watson, Michaelsen y Sharp (1991) en el 98\% de los casos, el desempeño del equipo supera el desempeño individual del mejor miembro del equipo. Teniendo en cuenta el origen ateórico del método, el investigador puede articularlo con referenciales teóricos, a fin de ampliar sus potencialidades. Así como, a 
partir de nuevas investigaciones, generar discursos tecnológicos inéditos que mejoren las técnicas del método.

En varias tecnologías aparecen destacadas la importancia dada por Michaelsen, Fink y Knight (2004) al desarrollo de equipos de aprendizaje y al tiempo mínimo necesario para que los grupos se conviertan en equipos (20 horas de interacción). El desarrollo de equipos es para estos autores tan importante como el aprendizaje, en cierto sentido, el desarrollo de equipos es una etapa necesaria para el aprendizaje. Consideramos que las tecnologías que generan las técnicas - y en cierta medida las tareas - relacionadas al desarrollo de equipos tienen raíces en el contexto de enseñanza universitaria de gestión y negocios. Posteriormente, su desarrollo histórico ocurrió en disciplinas del área de salud (Michaelsen, Parmelee, McMahon, Levine, \& Billings, 2007). Las dos áreas, históricamente, valorizan el trabajo en equipo mucho más que en disciplinas como la Física, Química o Matemática. Otra influencia de esas áreas en la construcción de la praxeología del Team-Based Learning puede ser vista en la técnica en la cual el profesor necesita elaborar problemas de toma de decisiones que contengan una única solución, como mejor selección para el gerenciamiento de un negocio o un mejor diagnóstico médico para un paciente enfermo.

El tiempo necesario para la formación de equipos de aprendizaje es una característica fundamental del TBL. Por ello, a diferencia de otros métodos, cuyas tareas y técnicas pueden ser evaluadas en pocas clases (e.g. Peer Instruction, Just-inTime Teaching), la adopción del TBL exige una planificación a largo plazo y una mayor modificación de las tareas y técnicas tradicionales. Podríamos decir que no tiene sentido pensar en aplicar TBL en una sola clase. Observemos que una interpretación de la praxis del TBL, sin el logos, puede dar como resultado modificaciones en las técnicas del método que lo descaracterizarían, como, por ejemplo, modificaciones constantes de los grupos de estudiantes a lo largo de un semestre o el año lectivo.

$\mathrm{Al}$ contrario de un abordaje del tipo "aprenda con la práctica", en que se parte de problemas de la realidad para comenzar la conceptualización, en el TBL se asume que algún conocimiento conceptual básico sobre el tema en estudio es esencial para la resolución de problemas. Por lo tanto, en ciertos tipos de tareas (por ejemplo, $\mathbf{T}_{\mathrm{a} 2} \mathrm{y} \mathbf{T}_{\mathrm{a} 3} \mathrm{de}$ la Figura 5) los estudiantes trabajan con conceptos básicos para realizar los tipos de tareas subsecuentes que implican la aplicación de conceptos $\left(\mathbf{T}_{\mathrm{a} 6}-\right.$ resolver individualmente en casa problemas de aplicación de conceptos estudiados en la fase de preparación, y la $\mathbf{T}_{\mathbf{a} 7}$ - aplicar, fuera de la clase, los conceptos adquiridos en la fase de preparación).

Todas las tareas de contenido son evaluadas por el docente en términos de correcta o incorrecta, lo que contribuye a la calificación final del estudiante, constituyéndose así en una forma de incentivar la realización de actividades y la responsabilidad con el equipo. Así, por ejemplo, para las preguntas del Test de Preparación Individual $\left(\mathbf{T}_{\mathrm{a} 2}\right.$, $\boldsymbol{\tau}_{\mathrm{a} 2} \mathbf{y} \boldsymbol{\theta}_{\mathrm{a} 2}$ en la Figura 5), los alumnos son evaluados antes de recibir cualquier feedback referente a dudas en la lectura en casa $\left(\mathbf{T}_{\mathrm{a} 3}, \boldsymbol{\tau}_{\mathrm{a} 3} \mathbf{y} \boldsymbol{\theta}_{\mathrm{a} 3}\right.$ en la Figura 5). El estímulo a la lectura del material indicado es generado mediante la recompensa o punición dada por la nota 
dada a los aciertos y errores en el Test de Preparación Individual. Lo mismo ocurre con el incentivo a la interposición de recurso, en el cual sólo los alumnos del equipo que lo redactó son recompensados con nota, y en el Test de Preparación en equipo, cuya manera de aumentar la cohesión de los grupos es recompensando su desempeño. En palabras de Fink (2004, p. 58), "la manera más obvia de incentivar a los miembros a dedicar tiempo y energía al trabajo en grupo es incluyendo el desempeño del grupo en el sistema de evaluación". Identificamos esa característica como pertenecientes a teorías (implícitas) behavioristas.

\footnotetext{
$\mathrm{T}_{\mathrm{a} 2} \quad$ Prepararse individualmente, en el aula para la resolución de problemas en equipo.

$\boldsymbol{\tau}_{\mathrm{a} 2}$ Responder, individualmente, las preguntas de un test conceptual de respuestas múltiples (Test de Preparación Individual) de preguntas cortas y directamente relacionadas con el material leído.

$\boldsymbol{\theta}_{\text {a2 }}$ Las respuestas del alumno al Test de Preparación Individual son evaluadas en términos de conocimiento, sumando para la nota final del estudiante, lo que incentiva a responsabilizarse por su propio estudio y a dedicarse a la lectura en casa.

Las preguntas son cortas y directas para evaluar el conocimiento básico del alumno respecto al material leído, o sea, deben contener datos fácilmente identificados en el libro, sin entrar en problemas complejos de toma de decisiones.

Esa actividad tiene como objetivo preparar al estudiante para la discusión y resolución de problemas de aplicación.
}

$\mathbf{T}_{\mathbf{a} 3}$ Prepararse colaborativamente, en clase, para la resolución de problemas de aplicación en equipo

$\tau_{\text {a3 }}$ Responder, en equipo, el mismo test resuelto individualmente (Test de Preparación en equipo), discutiendo con los colegas hasta lograr un consenso respecto a la respuesta correcta, marcándola en un cartel de corrección instantánea.

$\boldsymbol{\theta}_{\text {a3 }} \mathrm{Al}$ discutir las mismas cuestiones del Test de Preparación Individual con los colegas, se estimula la colaboración y, consecuentemente, el desarrollo de equipos de aprendizaje. Son necesarias por lo menos 20 horas de interacción para que un grupo se convierta en un equipo.

El Test de Preparación en equipo también es evaluado en términos de conocimiento, lo que hace que el estudiante sea incentivado por sus pares a prepararse antes de la clase, haciéndose responsable del aprendizaje de sus colegas. Una manera de aumentar la cohesión del grupo es recompensarlo por su desempeño.

Los cartones proporcionan un feedback instantáneo que posibilita, en caso de error, discusiones respecto de las dificultades de comprensión.

Esta actividad tiene como objetivo preparar al estudiante para la discusión y resolución de problemas de aplicación.

Figura 5. Algunos elementos del Team-Based Learning en términos de Tipos de tarea (T), Técnicas $(\tau)$ y Tecnologías $(\theta)$, que se describen en el topos del alumno (señalado por el índice "a")

Como ya fue señalado anteriormente, el origen del Team-Based Learning es ateórico, por lo menos explícitamente. O sea, el método fue desarrollado en base a la experiencia práctica y los conocimientos que los autores tenían acerca de su disciplina de origen (gestión y negocios) y de investigaciones en el área. Es natural que, a pesar 
de ser innovador, en contextos educacionales más conservadores, el método mantenga algunas características tradicionales, pues necesita, a priori, adaptarse a las condiciones institucionales normalmente sustentadas en una pedagogía dominante de naturaleza conductista. Se puede observar que, usando el análisis praxeológico, podemos distinguir los aspectos del método que poseen ese sesgo conductista e adaptarlos con otra visión teórica.

Para que el investigador conozca previamente las características del método, obtenidas a través del análisis praxeológico, lo ayuda a comprender experiencias exitosas o infructuosas del método. Tal comprensión puede ser ayudada por el análisis de los cambios en la práctica y en los discursos tecnológicos que las sostienen, explicitados en la elaboración de las Organizaciones de Métodos de Enseñanza. Además, el investigador, a partir de la organización de referencia, puede informar a los interesados en adoptar el método acerca de lo que puede o no ser modificado en TBL, sin reducir sus potencialidades.

Supongamos que quisiéramos usar TBL en el contexto de una disciplina de física básica con unos 40 estudiantes en una universidad pública brasileña sin la ayuda de monitores como los Teaching Assistants (TA) de las universidades estadounidenses. Esto podría complicar la tarea del profesor de realizar pequeñas presentaciones orales, teniendo en cuenta las dificultades de los estudiantes, ya que en TBL original, la técnica sugerida es evaluar las respuestas de los estudiantes en el Test de Preparación en equipo, que originalmente se realizó con la ayuda de monitores. Una posible modificación, a partir de los elementos del método Just-in-Time Teaching (Novak et al., 1999), es pedir que los estudiantes, además de leer antes de la clase, como ya se proporcionó en TBL, respondan tres preguntas relacionadas a la lectura, cuyas respuestas deben enviarse, antes de las clases, al profesor. Dos de las preguntas abordan los conceptos que se estudian y una de ellas evalúa la comprensión y el compromiso del estudiante con la actividad. Con las respuestas, el profesor puede preparar sus exposiciones basándose en las principales dificultades de los aprendices. Esos cambios, además de posibilitar que el profesor ejecute el tipo de tarea mencionado en un contexto en que da clase solo, también se justifica por la posibilidad que el alumno identifique lagunas en su propio entendimiento y, con eso, consiga orientar su propio proceso de aprendizaje. En TBL original, el estudiante se enfrenta a preguntas solo en el aula. Las modificaciones mencionadas en términos praxeológicos se muestran en la Figura 6.

Otra posible modificación, basada en concepciones constructivistas de la enseñanza, podría ser la transformación de los elementos conductuales (punitivos) presentes en OME de referencia de TBL. Originalmente, la evaluación del Test de Preparación individual se basa en la corrección de las respuestas y busca, a partir de la nota, animar a los estudiantes a prepararse para la clase $\left(\boldsymbol{\theta}_{\mathrm{a} 2}\right.$ de la Figura 5). Podemos realizar la evaluación de la actividad en términos de participación y esfuerzo en la actividad ( $\boldsymbol{\theta}_{\mathrm{a} 2}$ en la Figura 7). Para esto, en cada respuesta se puede exigir una justificación para que el alumno demuestre el razonamiento y exprese las dudas ( $\boldsymbol{\tau}_{\mathrm{a} 2}^{\prime}$ en la Figura 7$)$. 
Así el estudiante no se siente inhibido para cometer errores y se siente incentivado para intentar responder.

Es posible notar que las modificaciones presentadas no descaracterizan el método, sino que lo potencializan.

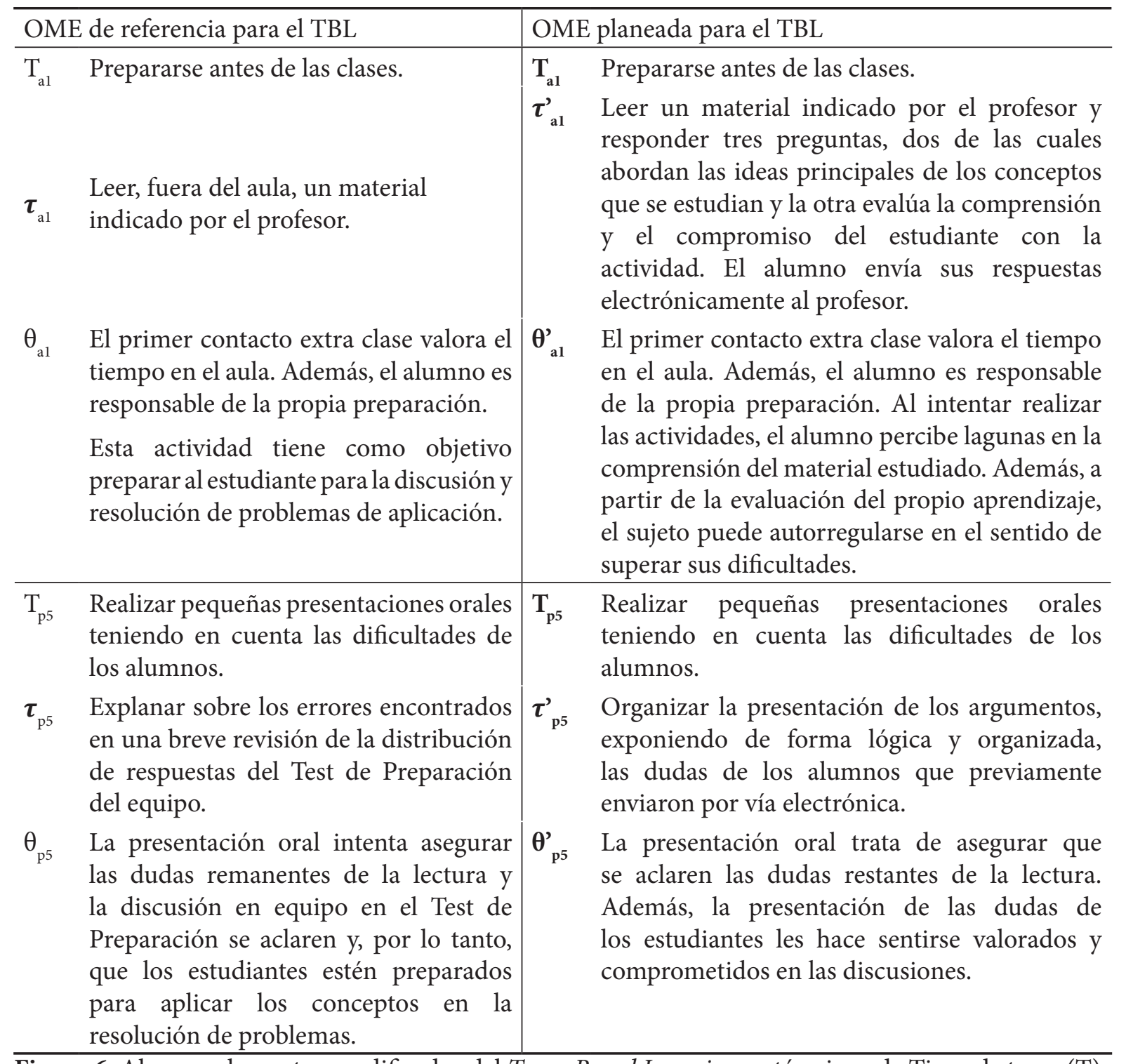

$\overline{\text { Figura 6. Algunos elementos modificados del Team-Based Learning en términos de Tipos de tarea (T), }}$ Técnicas $(\tau)$ y Tecnologías $(\theta)$, que se describen en topos del alumno (señalado por el índice "a") y del profesor (señalado por el índice "p"). El símbolo ", " representa una modificación en el elemento de la praxeología de referencia 
$\mathbf{T}_{\mathrm{a} 2}$ Prepararse individualmente, en el aula para la resolución de problemas en equipo.

$\boldsymbol{\tau}{ }_{\text {a2 }} \quad$ Responder individualmente a un test conceptual de selección múltiple con preguntas que impliquen reflexión (Test de Preparación individual). Se debe dar una justificación para cada respuesta marcada en el test.

$\boldsymbol{\theta}{ }^{2} \quad$ Las respuestas del alumno a la prueba de preparación individual se evalúan en términos de razonamiento demostrado y participación en la actividad, por lo que no se le impide cometer errores y se le recomienda tratar de responder. Al justificar las respuestas, el estudiante reflexiona sobre su propia comprensión y crea argumentos para discutir con sus compañeros de equipo el Test de Preparación en equipo.

Las preguntas del test fomentan la reflexión del estudiante sobre el material leído, que no requiere solo la memorización de lo que está en el texto.

Figura 7: Algunos elementos modificados del Team-Based Learning en términos de Tipos de tarea $(\mathrm{T})$, Técnicas $(\tau)$ y Tecnologías $(\theta)$, que se describen en topos del alumno (señalado por el índice "a"). El símbolo “ " " representa una modificación en el elemento de la praxeología de referencia.

\section{Consideraciones finales}

Añadimos a la amplia gama de posibilidades de aplicación de la Teoría Antropológica de lo Didáctico (TAD), la relectura de los métodos de enseñanza. Usualmente vistos como meras técnicas a disposición del profesor, los métodos de enseñanza, a partir de una perspectiva antropológica, son vislumbrados como un cuerpo de conocimiento social e históricamente legitimado y sensible a transformaciones, cuya composición puede ser descripta y analizada en términos de una Organización Praxeológica. A partir de ello, destacamos que es posible construir una praxeología para cualquier método de enseñanza, lo que brinda mayor claridad al investigador respecto a las partes que lo componen y sus justificaciones. Para sustentar nuestra propuesta, desarrollamos una praxeología de referencia para el método Team-Based Learning.

La perspectiva de la TAD para la investigación sobre métodos de enseñanza posibilita nuevos caminos para el área, permitiendo disminuir la brecha entre investigación y práctica, en la medida que el análisis de las especificidades de los métodos de enseñanza, presentadas y ejemplificadas en este artículo, proporciona detalles al investigador y al profesor que pueden facilitar la institucionalización de un método específico en condiciones ecológicas distintas a aquellas expuestas en el contexto de la investigación empírica. Esto se puede lograr cuando los discursos tecnológicos que justifican / generan / explican las técnicas propuestas en el método de enseñanza se explicitan, convirtiéndose así en objeto de escrutinio y diligencia. Además de esa aplicación en el análisis praxeológico de los métodos de enseñanza, que contribuyen a la construcción de un puente entre investigación empírica y práctica pedagógica, vislumbramos otras potencialidades que describimos a continuación.

El análisis praxeológico de los métodos de enseñanza posibilita la investigación de sus transposiciones, esto es, el estudio de cómo éstos se transforman o cómo transitan interinstitucionalmente. Al comparar las organizaciones de referencia, planeada e implementada, el investigador puede comprender cómo un cuerpo de conocimientos 
proveniente de la investigación empírica llega a la práctica de una institución particular.

Se pueden establecer diálogos entre teorías científicas y métodos de enseñanza, así como crear nuevos métodos fundamentados teóricamente. En el primer caso, el investigador compara los discursos que fundamentan un referencial con aquellos que justifican las técnicas de un método. A continuación, se modifican las tecnologías del método para que sean coherentes con la fundamentación teórica escogida. Tal modificación en las tecnologías genera alteraciones en la praxis del método que pueden potenciarlo. Para crear nuevos métodos de enseñanza, el investigador, a partir de un referencial teórico, establece algunos principios, en la forma de discursos tecnológicos, que lo ayudan a determinar las técnicas y los tipos de tareas. Es posible también establecer diálogos entre diferentes métodos de enseñanza, describiéndolos en términos de organizaciones praxeológicas y posteriormente, articulando sus partes.

Por último, el tipo de análisis que proponemos puede ser útil para la evaluación del impacto de las innovaciones didácticas, lo cual es determinante para su legitimación institucional. Al construir una praxeología del método de enseñanza, el investigador puede analizarla a fin de entender qué es lo relevante para ser evaluado y cómo realizarlo. Por ejemplo, un método como el Team-Based Learning, anteriormente analizado, justifica gran parte de sus técnicas en base a la idea de que es importante para el desarrollo de equipos de aprendizaje. Desde esa perspectiva, sería coherente analizar si a lo largo de un período, los grupos se transforman en equipos. Aún más, la praxeología del TeamBased Learning ayuda al investigador a determinar cómo puede realizarse ese análisis. Él podría, por ejemplo, utilizar los indicadores de evaluación entre colegas (preparación extra clase, contribución en las discusiones y trabajos, incentivo para que los colegas contribuyan con sus ideas y flexibilidad frente a los conflictos y desacuerdos) para determinar las dimensiones de análisis.

Además del campo de la investigación científica, la Organización Praxeológica como herramienta analítica puede ser aplicada en la formación de profesores, los cuales pueden, en régimen de observación o autoevaluación de la práctica, comparar los métodos de enseñanza originales com aquellos implementados e intentar comprender las modificaciones y, en qué medida, ellos pueden incorporar o distorsionar una idea originalmente promisoria. Nuestra propuesta le permite al profesor analizar y generar conocimiento sobre su propia práctica, dando forma a su conocimiento de enseñanza y, por lo tanto, mejorando el diálogo con el conocimiento en el campo de la investigación en educación de ciencias, ya sea por contraste (teórico-práctico) o complementación del conocimiento. Superar, por lo tanto, las barreras a veces impuestas por un modelo vertical entre la academia y la escuela.

\section{Agradecimientos}

Agradecemos a los árbitros de este artículo y al profesor Leonardo Albuquerque Heidemann (IF-UFRGS) por sus comentarios y sugerencias que contribuyeron a la mejora del trabajo. También agradecemos a la profesora 
Marta Pesa (Universidad Nacional de Tucumán) y al estudiante de doctorado en Enseñanza de Física en la UFRGS Elkin Adolfo Vera Rey. Ives Solano Araujo agradece al $\mathrm{CNPq}$ por la beca de productividad de investigación otorgada. Este trabajo se realizó con el apoyo de la Coordinación de Mejoramiento del Personal de Educación Superior - Brasil (CAPES) - Código de Financiamiento 001.

\section{Referencias}

Achiam, M. F. (2013). A Content-oriented Model for Science Exhibit Engineering. International Journal of Science Education, Part B, 3(3), 214-232. http://doi.org/10.1080 /21548455.2012.698445

Achiam, M., Lindow, B. E. K., \& Simony, L. (2019). Was Archaeopteryx able to fly? Authentic palaeontological practices in a museum programme. Educaçao Matemática Pesquisa, 21(4), 112-126.

Araujo, I. S., \& Mazur, E. (2013). Instrução pelos colegas e ensino sob medida: uma proposta para o engajamento dos alunos no processo de ensino-aprendizagem de Física. Caderno Brasileiro de Ensino de Física, 30(2), 362-384. http://doi.org/10.5007/21757941.2013v30n2p362

Barbé, J., Bosch, M., Espinoza, L., \& Gascón, J. (2005). Didactic restrictions on the teacher's practice: The case of limits of functions in Spanish high schools. Educational Studies in Mathematics, 59(1-3), 235-268. http://doi.org/10.1007/0-387-30451-7_9

Barbé, J., Espinoza, L., \& Gellert, U. (2017). El empobrecimiento matemático de las propuestas de enseñanza de Física en los textos oficiales de secundaria. Enseñanza de Las Ciencias, 35(1), 71-88. http://dx.doi.org/10.5565/rev/ensciencias.1730

Barquero, B., Bosch, M., \& Gascón, J. (2011). Los recorridos de estudio e investigación y la modelización matemática en la enseñanza universitaria de las ciencias experimentales. Enseñanza de las Ciencias, 29(3), 339-352.

Bergmann, J., \& Sams, A. (2012). Flip your classroom: Reach every student in every class every day. Washington: International Society for Technology in Education.

Bosch, M., Gascón, J., \& Trigueros, M. (2016). Dialogue between theories interpreted as research praxeologies: The case of APOS and the ATD. Educational Studies in Mathematics, 95(1), 39-52. http://doi.org/10.1007/s10649-016-9734-3

Bueno, J. P. P., \& Marandino, M. (2017). The notion of praxeology as a tool to analyze educational process in science museums. In K. Hahl, K. Juuti, J. Lampiselkä, A. Uitto, \& J. Lavonen (Orgs.), Cognitive and affective aspects in science education research, Contributions from science education research, Vol. 3, (pp. 339-355). Cham: Springer International Publishing. 
Cardelli, J. (2004). Reflexiones críticas sobre el concepto de Transposición Didáctica de Chevallard. Cuadernos de Antropología Social, (19), 49-61.

Caillot, M. (1996). La Théorie de la transposition didactique est-elle transposable? In: C. Raisky, \& C. Caillot (Org.), Au-delá des didactiques, le didactique: Débats autor de concepts Fédérateurs (pp. 19-35). France: De Boeck \& Larcier.

Chervel, A. (1990). As histórias das disciplinas escolares. Reflexões sobre um domínio de pesquisa. Teoria \& Educação, (2), 177-229.

Chevallard, Y. (1991). La transposición didáctica: Del saber sabio al saber enseñado. Buenos Aires: Aique.

Chevallard, Y. (1999). El análisis de las prácticas docentes en la teoría antropológica de lo didáctico. Recherches em Didactique des Mathématiques, 19(2), 221-266.

Chevallard, Y. (2000). Conceitos fundamentais da didáctica: As perspectivas trazidas por uma abordagem antropológica. In J. Brun. (Org.). Didáctica das matemáticas (pp. 115-152). Lisboa: Instituto Piaget.

Chevallard, Y., Bosch, M., \& Gascón, J. (2001). Estudar matemáticas - O elo perdido entre o ensino e a aprendizagem. Porto Alegre: Artmed.

Chevallard, Y. (2013). Enseñar Matemáticas en la Sociedad de Mañana: Alegato a Favor de un Contraparadigma Emergente. Journal of Research in Mathematics Education REDIMAT - Journal of Research in Mathematics Education, 2(2), 161-182.

Chevallard, Y. (2019). Introducing the Anthropological Theory of the Didactic: An attempt at a Principled Approach. Hiroshima Journal of Mathematics Education, (12), 71-114.

Contreras, J. A. (2012). A autonomia de professores (2a. ed.). São Paulo: Cortez.

Corica, A. R., \& Otero, M. R. (2011). Análisis de la dinámica de estudio en un curso universitario de matemática. In Un panorama de la TAD. III Congreso Internacional sobre la TAD (pp. 605-625).

Corica, A. R., \& Otero, M. R. (2019). Análisis de la Gestión de un Dispositivo Didáctico por un Estudiante de Profesorado en Matemática. Bolema: Boletim de Educação Matemática, 33(63), 226-247. https://doi.org/10.1590/1980-4415v33n63a11

Crouch, C. H., \& Mazur, E. (2001). Peer Instruction: Ten years of experience and results. American Journal of Physics, 69(9), 970. http://doi.org/10.1119/1.1374249

Dancy, M., \& Henderson, C. (2010). Pedagogical practices and instructional change of physics faculty. American Journal of Physics, 78(10), 1056-1063. http://doi. org/10.1119/1.3446763

Douglas, M. (1986). How institutions think. London: Routledge and Kegan Paul. 
El-Hani, C. N., \& Greca, I. M. (2011). Participação em uma comunidade virtual de prática desenhada como meio de diminuir a lacuna pesquisa-prática na educação em biologia. Ciência \& Educação (Bauru), 17(3), 579-601. http://doi.org/10.1590/S151673132011000300005

Espinosa, T., Araujo, I. S., \& Veit, E. A. (2016). Aprendizagem Baseada em Equipes (Team-Based Learning): um método ativo para o Ensino de Física. Caderno Brasileiro de Ensino de Física, 33(3), 962-986. https://doi.org/10.5007/2175-7941.2016v33n3p962

Espinosa, T. (2019). Adoção de inovações didáticas no ensino universitário de física na perspectiva de transposições praxeológicas. (Tese de Doutorado em Ensino de Física). Programa de Pós-Graduação em Ensino de Física da Universidade Federal do Rio Grande do Sul, Porto Alegre.

Foote, K. T. (2016). Curriculum development in studio-style university physics and implications for dissemination of research-based reforms. Physical Review Physics Education Research,12(1),1-18.http://doi.org/10.1103/PhysRevPhysEducRes.12.010127

Hake, R. (1998). Interactive-engagement versus traditional methods: A six-thousandstudent survey of mechanics test data for introductory physics courses. American Journal of Physics, 66(1), 64-74. http://dx.doi.org/10.1119/1.18809

Henderson, C., \& Dancy, M. H. (2008). Physics faculty and educational researchers: Divergent expectations as barriers to the diffusion of innovations. American Journal of Physics, 76(1), 79-91. http://doi.org/10.1063/1.2177045

Henderson, C., \& Dancy, M. H. (2009). Impact of physics education research on the teaching of introductory quantitative physics in the United States. Physical Review Special Topics - Physics Education Research, 5(2), 020107. http://doi.org/10.1103/ PhysRevSTPER.5.020107

Khatri, R., Henderson, C., Cole, R., Froyd, J. E., Friedrichsen, D., \& Stanford, C. (2017). Characteristics of well-propagated teaching innovations in undergraduate STEM. International Journal of STEM Education, 4(1), 1-10. http://doi.org/10.1186/s40594017-0056-5

Leite, M. S. (2004). Contribuições de Basil Bernstein e Yves Chevallard para a discussão do conhecimento escolar. (Dissertação de Mestrado em Educação). Departamento de Educação do Centro de Teologia e Ciências Humanas da PUC-Rio, Rio de Janeiro.

Marandino, M., Bueno, J., Kristel, F. L., \& Oliveira, A. (2016). Os usos da Teoria da Transposição Didática e da Teoria Antropológica do Didático para o estudo da educação em museus de ciências. Revista Labore Em Ensino de Ciências, 1(1), 69-97.

Mazur, E. (2015). Peer Instruction: A revolução da aprendizagem ativa. Porto Alegre: Penso. 
McIntyre, D. (2005). Bridging the gap between research and practice. Cambridge Journal of Education, 35(3), 357-382. http://doi.org/10.1080/03057640500319065

Michaelsen, L. K., Knight, A. B., \& Fink, L. D. (2004). Team-Based Learning: A transformative use of small groups in college teaching. Sterling: Stylus.

Michaelsen, L. K., Parmelee, D. X, McMahon, K. K., Levine, R. E., \& Billings, D. M. (2007). Team-Based Learning for Health Professions Education: A guide to using small groups for improving learning. VA: Stylus Publishing, LLC.

Michaelsen, L. K., \& Sweet, M. (2011). Team-based learning. New Directions for Teaching and Learning, (128), 41-51.

Miretzky, D. (2007). A view of research from practice: Voices of teachers. Theory into Practice, 46(4), 272-280. http://doi.org/10.1080/00405840701593857

Novak, G. M., Patterson, E. T., Gavrin, A., \& Christian, W. (1999). Just-In-Time Teaching: Blending active learning and web technology. Saddle River: Prentice Hall.

Rogers, E. M. (2003). Diffusion of Innovations (5. ed.). New York: Free Press of Glencoe. Rudolph, A. L., Lamine, B., Joyce, M., Vignolles, H., \& Consiglio, D. (2014). Introduction of interactive learning into French university physics classrooms. Physical Review Special Topics - Physics Education Research, 10(1), 1-18. http://doi.org/10.1103/ PhysRevSTPER.10.010103.

Schivani, M., Brockington, G., \& Pietrocola, M. (2013). Aplicações da Robótica no Ensino de Física: Análise de Atividades numa Perspectiva Praxeológica. Revista de Educacion En Ciencias, Journal of Science Education, 14, 32-36.

Trigueros, M., \& Martinez-Planell, R. (2015). Las funciones de dos variables: Análisis mediante los resultados del diálogo entre la teoría APOS y la TAD. Enseñanza de Las Ciencias, 33(2), 157-171. http://dx.doi.org/10.5565/rev/ensciencias. 1520

Watson, W. E., Michaelsen, L. K., \& Sharp, W. (1991). Member competence, group interaction, and group decision making: A longitudinal study. Journal of Applied Psychology, 76(6), 803-809. http://dx.doi.org/10.1037/0021-9010.76.6.803

Wijayanti, D. (2019). Linking proportionality of arithmetic, algebra and geometry domains in Indonesian lower secondary textbooks. Educação Matemática Pesquisa, 21(4), 74-84.

Wijayanti, D., \& Winsløw, C. (2018). Mathematical practice in textbooks analysis: Praxeological reference models, the case of proportion. Journal of Research in Mathematics Education, 6(3), 307. https://doi.org/10.17583/redimat.2017.2078

Winsløw, C. (2011). Anthropological theory of didactic phenomena: some examples and principles of its use in the study of mathematics education. Un Panorama de TAD, CRM Docume (January 2011), 117-138. 
Tobias Espinosa

https://orcid.org/0000-0002-6958-8274

Universidade Federal do Rio Grande

Instituto de Matemática, Estatística e Física Santo Antônio da Patrulha, Rio Grande do Sul, Brasil tobiasespinosa@furg.br

Ives Solano Araujo

(ㄱ) https://orcid.org/0000-0002-3729-0895 Universidade Federal do Rio Grande do Sul

Instituto de Física

Porto Alegre, Rio Grande do Sul, Brasil ives@if.ufrgs.br

\section{Eliane Angela Veit}

${ }^{\circ}$ https://orcid.org/0000-0002-2406-3415 Universidade Federal do Rio Grande do Sul Instituto de Física Porto Alegre, Rio Grande do Sul, Brasil eav@if.ufrgs.br

Submetido em 15 de abril de 2019

Aceito em 15 de agosto de 2019

Publicado em 11 de setembro de 2019 